\section{Delamanid als Add-on bei multiresistenter Tuberkulose}

Groote-Bidlingmaier F et al. Efficacy and safety of delamanid in combination with an optimized background regimen for treatment of multidrug-resistant tuberculosis: a multicentre, randomized, double-blind, placebo-controlled, parallel-group phase 3 trial. Lancet Respir Med 2019; PMID: 30630778. doi:10.1016/S22132600(18)30426-0

Laut WHO erfordert die Tuberkulose dringend die weitere Erforschung von wirksamen Therapien gegen multiresistente Stämme von M. tuberculosis (MDR). Delamanid ist seit 2014 in Europa zur Therapie der MDR-Tbc unter bestimmten Bedingungen zugelassen. Ob Delamanid als Add-onWirkstoff zu einer leitliniengerechten üblichen Tbc-Therapie Vorteile bei der Behandlung von Patienten mit MDRTbc hat, wollten die Autoren in dieser Phase-3-Studie zeigen.

Zwischen 2011 und 2013 wurden 511 erwachsene Patienten (bis 69 Jahre) mit multiresistenter Tbc in 17 Ländern für diese doppelblinde, placebokontrollierte Phase-3-Studie rekrutiert. Als Einschlusskriterium galt das Vorliegen mindestens einer für $M$. tuberculosis positiven Sputumkultur bei Nachweis einer Resistenz gegenüber Isoniazid und Rifampicin. Ausgeschlossen wurden u.a. Patienten mit schweren Begleitkrankheiten, Leberoder Nierenfunktionsstörungen, kardiovaskulären Krankheiten, extremem Untergewicht oder Kontraindikationen für die geplante Therapie. In einem 2:1-Design erhielten 341 von ihnen Delamanid oral in einer Dosis von 2-mal $100 \mathrm{mg}$ tgl. für 2 Monate und anschließend 1-mal $200 \mathrm{mg}$ tgl. für weitere 4 Monate. Den übrigen 170 Patienten wurde Placebo zugeschrieben; alle wurden wie von der WHO und nationalen Leitlinien empfohlen ansonsten individuell optimal gegen Tuberkulose behandelt (optimised background regimen). Alle 511 Patienten wurden in die Analyse der Sicherheit eingeschlossen. Da sich im Verlauf herausstellte, dass sich bei einigen Patienten M. tuberculosis doch nicht nachweisen ließ, keine Multiresistenz vorlag oder ein Screening nicht erfolgen konnte, bildeten die Autoren eine modifizierte Intention-to-treat-Population zur Analyse der Effektivität. Diese beinhaltete 226 Patienten in der Interventionsgruppe und 101 in der Placebogruppe; 32 und 16 von ihnen waren HIV-positiv. Fast 95\% nahmen mindestens 22 Wochen lang die Medikation ein.

Der primäre Endpunkt - die Zeit bis zur Sputumkulturkonversion in einem Beobachtungszeitraum von 6 Monaten - unterschied sich mit 51 Tagen (Median) unter Delamanid und 57 Tagen unter Placebo nicht zwischen den Gruppen (Hazard Ratio 1,17; $p=0,2157$ ). Bei den sekundären Endpunkten wie weiterhin negative Sputumkultur zwischen 18 und 30 Monaten, Beurteilung des Therapieerfolgs durch den behandelnden Arzt u.a. ergaben sich ebenfalls keine Unterschiede. Allerdings zeigten zwei Sensitivitätsanalysen einen Vorteil für Delamanid.

Die Rate an ernsten therapiebedingten unerwünschten Wirkungen war mit 26,1\% (Delamanid) und 27,6\% (Placebo) gleich hoch. Bei 0,9\% der behandelten Patienten entwickelte sich eine Resistenz gegenüber Delamanid. Die Studie wurde vom Hersteller von Delamanid gesponsert.

\section{FAZIT}

In dieser laut Autoren ersten randomisierten placebokontrollierten Phase-3-Studie bei MDR-Tbc führte Delamanid in der Intention-to-treatPopulation nicht zu einer schnelleren Sputumkulturkonversion im Vergleich zu Placebo. Dennoch empfehlen die Autoren, Delamanid als Therapieoption noch genauer zu untersuchen, denn neben den beiden positiven Sensitivitätsanalysen erlangte die Placebogruppe hier eine außergewöhnlich hohe Heilungsrate von $78 \%$, was den Benefit von Delamanid möglicherweise verdeckt hat.

Dr. med. Susanne Meinrenken, Bremen 